\title{
Importance of Aromatic Content for Peptide/Single-Walled Carbon Nanotube Interactions
}

\author{
V. Zorbas, ${ }^{1}$ A. L. Smith, ${ }^{1}$ A. Ortiz-Acevedo, ${ }^{1}$ H. Xie, ${ }^{1,2}$ G. R. Dieckmann, ${ }^{1,2}$ R. K. Draper, ${ }^{1,2,3}$ R. H. \\ Baughman, ${ }^{1,2}$ I. H. Musselman ${ }^{1,2}$ \\ ${ }^{1}$ Department of Chemistry, University of Texas at Dallas, Richardson, TX 75083 \\ ${ }^{2}$ NanoTech Institute, University of Texas at Dallas, Richardson, TX 75083 \\ ${ }^{3}$ Department of Molecular and Cell Biology, University of Texas at Dallas, Richardson, TX 75083
}

Biological materials, such as peptides, are well known in nature for having unparalleled tailorability, specificity, and ability to self assemble into a wide variety of complex functional structures. Peptides have opened new possibilities for the dispersion, separation and manipulation of singlewalled carbon nanotubes (SWNTs). We have previously demonstrated that a designed peptide, denoted nano-1, coats and debundles SWNTs and promotes the assembly of these coated SWNTs into novel hierarchical structures via peptide-peptide interactions [1,2]. Nano-1 folds into an amphiphilic $\alpha$-helix, in which apolar residues in the peptide primary sequence occupy the $a$ and $d$ positions in the repeating heptad, denoted $(a, b, c, d, e, f, g)_{\mathrm{n}}$ forming a hydrophobic face and more polar residues occupy the $b, c, e, f$, and $g$ positions forming a hydrophilic face [1]. The aim of this study is to better understand how the aromatic content in the peptide affects the dispersion of SWNTs. The importance of aromaticity in amphiphilic helical peptides was tested using a series of designed peptides that vary in the number of aromatic residues on the hydrophobic surface of the helix. The peptides $\left(\mathrm{V}_{a} \mathrm{~L}_{d}\right)_{4 / 4}$ (previously well-characterized peptide Coil $\mathrm{V}_{a} \mathrm{~L}_{d}$ [3]), nano-1 (also called $\left(\mathrm{V}_{a} \mathrm{~F}_{d}\right)_{4 / 4}$ in this manuscript), and $\left(\mathrm{F}_{a} \mathrm{~F}_{d}\right)_{4 / 4}$, have varying numbers of phenylalanine (Phe or F) residues in the $a$ and $d$ positions (0, 4, and 8, respectively) (Fig. 1).

Peptide/SWNT dispersions were generated using a sample preparation procedure in which a mixture of peptide solution $(1 \mathrm{~mL}, 100 \mu \mathrm{M})$ and solid SWNTs $(1.0-1.3 \mathrm{mg})$ were sonicated (1 min, $10 \mathrm{~W})$ and centrifuged (50,000 x g, $30 \mathrm{~min}$ ) yielding dispersions (supernatants), which were removed from the undispersed solid [2]. Atomic force microscopy (AFM) images were acquired of these peptide/SWNT dispersions (Fig 2). Optical spectroscopy and AFM measurements revealed that sequences which placed more aromatic amino acids in the $a$ and $d$ positions were more effective at dispersing individual SWNTs (Fig 3). Isolation of individual SWNTs was achieved with an amphiphilic peptide design containing four aromatic amino acids in the $d$ position $\left(\left(\mathrm{V}_{a} \mathrm{~F}_{d}\right)_{4 / 4}\right)_{\text {, }}$ although the addition of more aromatic amino acids in the $a$ position $\left(\left(\mathrm{F}_{a} \mathrm{~F}_{d}\right)_{4 / 4}\right)$ resulted in an increased amount of SWNTs that were dispersed. Without aromatic amino acids in the apolar face of the amphiphilic helix $\left(\left(\mathrm{V}_{a} \mathrm{~L}_{d}\right)_{4 / 4}\right)$, only small bundles of SWNTs can be suspended. Altogether, these data demonstrate that $\pi$-stacking interactions between aromatic residues of a peptide and the graphene surface of a SWNT play a principle role in peptide dispersion of SWNTs.

References

[1] G. R. Dieckmann et al., J. Am. Chem. Soc. 125, (2003) 1770.

[2] V. Zorbas et al., J. Am. Chem. Soc. 126, (2004) 7222.

[3] N. L. Ogihara et al., Prot. Sci. 8, (1999) 1400.

[4] The support of this research by the Robert A. Welch Foundation [AT-1326 (I.H.M.)] and Department of Homeland Security (V.Z.) is gratefully acknowledged. 


$$
\begin{array}{ll}
\left(\mathrm{V}_{a} \mathrm{~L}_{d}\right)_{4 / 4}-0 \text { Phe } & \text { AC-E VEALEKK VAALESK VQALEKK VEALEHG-CONH } \\
\left(\mathrm{V}_{a} \mathrm{~F}_{d}\right)_{4 / 4}-4 \text { Phe } & \text { AC-E VEAFEKK VAAFESK VQAFEKK VEAFEHG-CONH }{ }_{2} \\
\left(\mathrm{~F}_{a} \mathrm{~F}_{d}\right)_{4 / 4}-8 \text { Phe } & \text { AC-E FEAFEKK FAAFESK FQAFEKK FEAFEHG-CONH }{ }_{2}
\end{array}
$$

Figure 1. Sequences of amphiphilic helical peptides designed to test the role of aromatic residues on peptide/SWNT interactions. Each seven residue repeat represents one helix heptad. Peptides $\left(\mathrm{V}_{a} \mathrm{~L}_{d}\right)_{4 / 4},\left(\mathrm{~V}_{a} \mathrm{~F}_{d}\right)_{4 / 4}$ (i.e. nano-1), and $\left(\mathrm{F}_{a} \mathrm{~F}_{d}\right)_{4 / 4}$ have varying numbers of Phe residues in the $a$ and $d$ positions $(0,4$, and 8 , respectively).
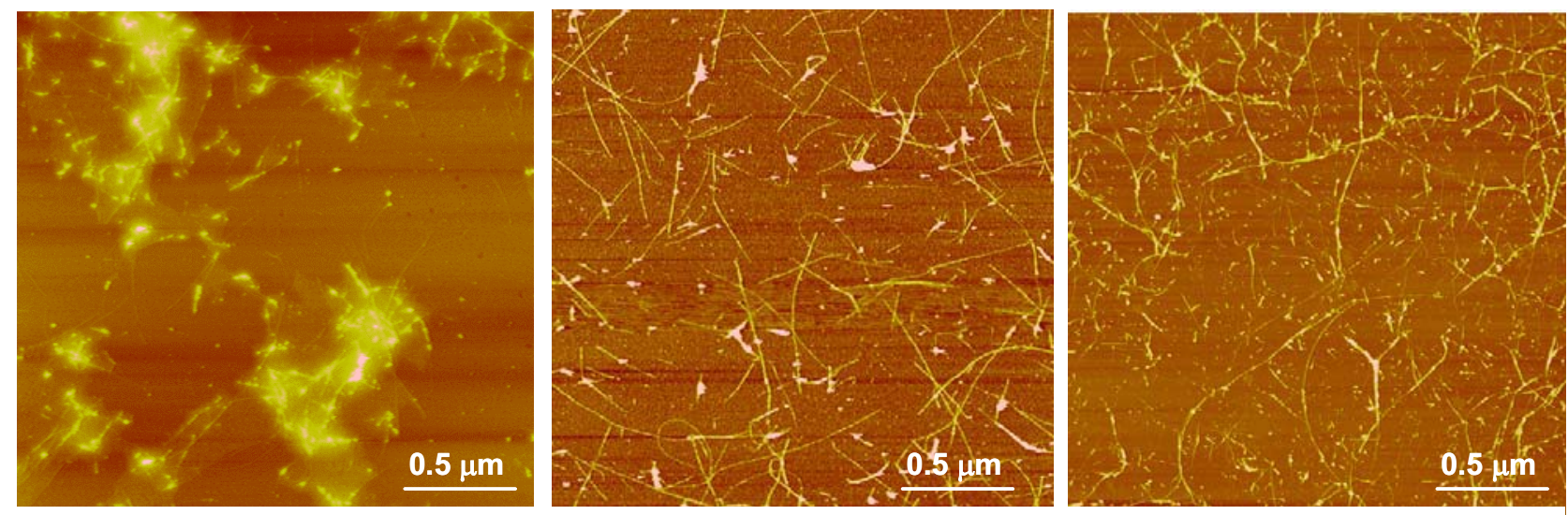

Figure 2. AFM images of (a) $\left(\mathrm{V}_{a} \mathrm{~L}_{d}\right)_{4 / 4} / \mathrm{SWNT}$ sample exhibiting short SWNTs on film and pools of peptide, and (b) $\left(\mathrm{V}_{a} \mathrm{~F}_{d}\right)_{4 / 4} / \mathrm{SWNT}$ and (c) $\left(\mathrm{F}_{a} \mathrm{~F}_{d}\right)_{4 / 4} / \mathrm{SWNT}$ samples both showing long, individual SWNTs.

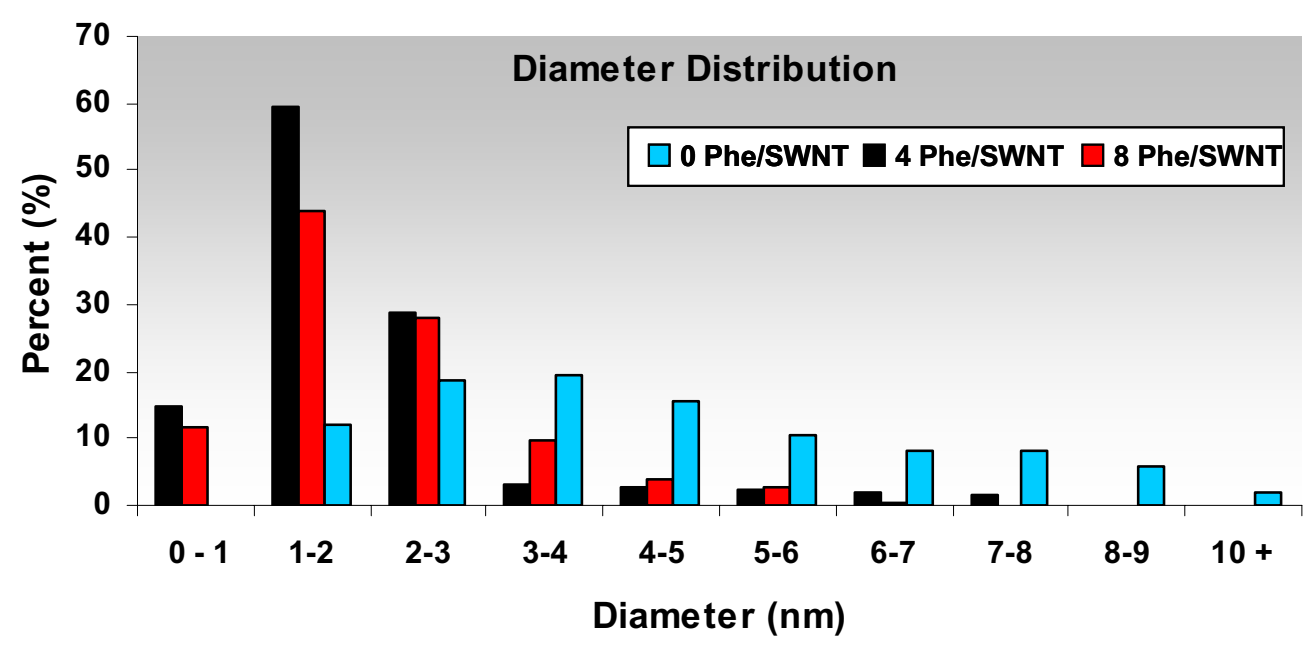

Figure 3. Diameter distributions (determined from AFM height measurements) of $\left(\mathrm{V}_{a} \mathrm{~L}_{d}\right)_{4 / 4}$, $\left(\mathrm{V}_{a} \mathrm{~F}_{d}\right)_{4 / 4}$, and $\left(\mathrm{F}_{a} \mathrm{~F}_{d}\right)_{4 / 4}$ dispersions of SWNTs. The diameters of $\left(\mathrm{V}_{a} \mathrm{~F}_{d}\right)_{4 / 4} / \mathrm{SWNT}$ and $\left(\mathrm{F}_{a} \mathrm{~F}_{d}\right)_{4 / 4} / \mathrm{SWNT}$ samples are indicative of debundled individual peptide-wrapped SWNTs, while the diameters of $\left(\mathrm{V}_{a} \mathrm{~L}_{d}\right)_{4 / 4} / \mathrm{SWNT}$ samples appear to represent small SWNT bundles. 